\title{
Characterization of leptospiral proteins that afford partial protection in hamsters against lethal challenge with Leptospira interrogans
}

Correspondence

Ana L. T. O. Nascimento tabet@butantan.gov.br

Received 16 April 2010 Accepted 10 June 2010

\author{
Marina V. Atzingen, ${ }^{1}$ Amane P. Gonçales, ${ }^{2}$ Zenaide M. de Morais, ${ }^{2}$ \\ Eduardo R. Araújo, ${ }^{3}$ Thales De Brito, ${ }^{3}$ Silvio A. Vasconcellos ${ }^{2}$ \\ and Ana L. T. O. Nascimento ${ }^{1,4}$ \\ ${ }^{1}$ Centro de Biotecnologia, Instituto Butantan, Avenida Vital Brazil 1500, 05503-900 São Paulo, SP, \\ Brazil \\ ${ }^{2}$ Laboratório de Zoonoses Bacterianas do VPS, Faculdade de Medicina Veterinária e Zootecnia, \\ USP, Avenida Prof. Dr Orlando Marques de Paiva 87, 05508-270 São Paulo, SP, Brazil \\ ${ }^{3}$ Instituto de Medicina Tropical, Departamento de Patologia, Faculdade de Medicina, USP, \\ Avenida Dr Enéas Carvalho de Aguiar 470, 05403-000 São Paulo, SP, Brazil \\ ${ }^{4}$ Interunidades em Biotecnologia, Instituto de Ciências Biomédicas, USP, Avenida Prof. \\ Lineu Prestes 1730, 05508-900 São Paulo, SP, Brazil
}

\begin{abstract}
Leptospirosis is a worldwide zoonosis caused by pathogenic Leptospira. The whole-genome sequence of Leptospira interrogans serovar Copenhageni together with bioinformatic tools allow us to search for novel antigen candidates suitable for improved vaccines against leptospirosis. This study focused on three genes encoding conserved hypothetical proteins predicted to be exported to the outer membrane. The genes were amplified by PCR from six predominant pathogenic serovars in Brazil. The genes were cloned and expressed in Escherichia coli strain BL21-SI using the expression vector pDEST17. The recombinant proteins tagged with $\mathrm{N}$-terminal $6 \times$ His were purified by metal-charged chromatography. The proteins were recognized by antibodies present in sera from hamsters that were experimentally infected. Immunization of hamsters followed by challenge with a lethal dose of a virulent strain of Leptospira showed that the recombinant protein rLIC12730 afforded statistically significant protection to animals (44\%), followed by rLIC10494 (40\%) and rLIC12922 (30\%). Immunization with these proteins produced an increase in antibody titres during subsequent boosters, suggesting the involvement of a T-helper 2 response. Although more studies are needed, these data suggest that rLIC12730 and rLIC10494 are promising candidates for a multivalent vaccine for the prevention of leptospirosis.
\end{abstract}

\section{INTRODUCTION}

Leptospirosis, an emerging infectious disease, is a worldwide zoonosis of human and veterinary concern. Caused by pathogenic spirochaetes of the genus Leptospira, the disease presents with greater incidence in tropical and subtropical regions (Bharti et al., 2003; Faine et al., 1999). The transmission of leptospirosis has been associated with the

Abbreviations: CD, circular dichroism; CDS, coding sequence; HRP horseradish peroxidase; LAg, leptospiral antigen; OMP, outer-membrane protein; Th, T helper.

The GenBank/EMBL/DDBJ accession numbers for the coding sequences of LIC10494, LIC12730 and LIC12922 are AAS69115, AAS71287 and AAS71474, respectively. The gene sequence accession numbers can also be accessed by the gene locus LIC number (Leptospira interrogans Copenhageni). exposure of individuals in close proximity to wild or farm animals (Faine et al., 1999; Levett, 2001). Lately, the disease has become prevalent in cities with sanitation problems and large populations of urban rodent reservoirs, which contaminate the environment through their urine (Ko et al., 1999). Fever, chills, headache and severe myalgias characterize the early phase of the disease. Progression to multi-organ system complications occurs in $5-15 \%$ of cases, with mortality rates of 5-40\% (Faine et al., 1999; Plank \& Dean, 2000).

Currently available veterinarian vaccines are based on inactivated whole-cell or membrane preparations of pathogenic leptospires. These types of vaccine confer protective responses through, although not exclusively, the induction of antibodies against leptospiral LPS (Adler 
\& de la Pena Moctezuma, 2010; de la Pena-Moctezuma et al., 1999). However, these vaccines do not induce longterm protection against infection and do not provide crossprotective immunity against leptospiral serovars not included in the vaccine preparation.

Outer-membrane proteins (OMPs) are attractive alternatives to whole-cell inactivated vaccines because of their antigenic conservation across leptospiral species and serovars (Cullen et al., 2003; Palaniappan et al., 2007). A number of transmembrane and lipoprotein OMPs are surface-exposed and expressed during infection of the mammalian host (Cullen et al., 2005; McBride et al., 2005). Several research groups have tested the protective characteristics of several recombinant protein vaccines, including leptospiral OMP OmpL1, lipoprotein LipL41 (Haake et al., 1999), haemolysis-associated protein 1/lipoprotein LipL32 (Branger et al., 2001; Seixas et al., 2007a, b), immunoglobulin-like (Lig) protein (Koizumi \& Watanabe, 2004; Palaniappan et al., 2006; Silva et al., 2007; Yan et al., 2009) and recombinant leptospiral putative OMPs (Chang et al., 2007). More recently, Yan et al. (2010) showed that putative leptospiral OMPs with an OmpA-like domain conferred partial protection against lethal infection with leptospira.

In the present work, we have described the cloning, expression, purification and characterization of three predicted leptospiral OMPs encoded by the genes LIC10494, LIC12730 and LIC12922, identified by bioinformatics tools in the genome sequence of Leptospira interrogans serovar Copenhageni. We evaluated the immune response promoted by these recombinant proteins in hamsters. Our results showed that the recombinant proteins encoded by the LIC12730 and LIC10494 genes afforded partial protection against lethal challenge with $L$. interrogans in hamsters. The immunoprotection conferred on these animals involved a T-helper 2 (Th2) response, as suggested by the increase in antibody titres during subsequent boosters. Although more studies are needed, our data suggest that these proteins may serve as subunit vaccine candidates.

\section{METHODS}

Leptospira strains and culture conditions. The non-pathogenic Leptospira biflexa (serovar Patoc strain Patoc 1) and the pathogenic $L$. interrogans (serovars Canicola strain Hond Utrechet IV, Copenhageni strain M-20, Grippotyphosa strain Moskva V, Hardjo strain Hardjoprajtino, Icterohaemorrhagiae strain RGA and Pomona strain Pomona), and the virulent $L$. interrogans serovar Pomona strain LPF and serovar Copenhageni strain Fiocruz L1-130, were cultured at $28{ }^{\circ} \mathrm{C}$ under aerobic conditions in liquid EMJH medium (Difco) with $10 \%$ rabbit serum, enriched with L-asparagine $(0.015 \%$, w/v), sodium pyruvate $(0.001 \%$, w/v $), \mathrm{CaCl}_{2}\left(0.001 \%\right.$, w/v), $\mathrm{MgCl}_{2}$ $(0.001 \%, \mathrm{w} / \mathrm{v})$, peptone $(0.03 \% \mathrm{w} / \mathrm{v})$ and meat extract $(0.02 \% \mathrm{w} / \mathrm{v})$ (Turner, 1970). The virulence of L. interrogans serovar Pomona strain LPF and serovar Copenhageni strain Fiocruz L1-130 was maintained by iterative passages in Golden Syrian hamsters (Faine et al., 1999). The animals were infected with $10^{4}$ leptospires and sacrificed after the appearance of symptoms, such as loss of weight and mobility ( $\sim 5$ days post-infection). Kidneys were removed and macerated and the leptospires were recovered through culture (Faine et al., 1999).

In silico identification and characterization of the protein. Putative coding sequences (CDSs) were selected from the L. interrogans serovar Copenhageni genome sequence (Nascimento et al., 2004a) based on their cellular localization prediction by PSORT (http://psort. nibb.ac.jp; Nakai \& Kanehisa, 1991; Nakai \& Horton, 1999) and Cello (http://cello.life.nctu.edu.tw; Yu et al., 2004, 2006). The SMART (http:// smart.embl-heidelberg.de; Letunic et al., 2006; Schultz et al., 1998) and PFAM (http://www.sanger.ac.uk/Software/Pfam; Finn et al., 2006) web servers were used to search for predicted functional and structural domains within the amino acids of the selected CDSs. The predicted lipobox sequence was evaluated using the LipoP (http://www.cbs.dtu. $\mathrm{dk} /$ services/LipoP; Juncker et al., 2003) and SpLip (Setubal et al., 2006) programs.

DNA isolation and PCR analysis. Leptospira cultures (L. interrogans serovars Canicola, Copenhageni, Grippotyphosa, Hardjo, Icterohaemorrhagiae and Pomona, and the non-pathogenic L. biflexa serovar Patoc) were harvested by centrifugation at $11500 \mathrm{~g}$ for $30 \mathrm{~min}$ and gently washed twice in sterile PBS. Genomic DNA was isolated from the pellets by the guanidine detergent lysing method using DNAzol Reagent (Invitrogen), according to the manufacturer's instructions. The DNA fragments were amplified using oligonucleotides designed according to $L$. interrogans serovar Copenhageni genome sequence (GenBank accession no. AE016823): LIC10494-F, 5'-CACCACTGCTAGGGCTGCAGAAA-3', and LIC10494-R1, 5' -GAATTGGTTTCGGTGGAAGTTGTAGTC-3'; LIC12730-F， 5'-CACCAGTTCTGACGGACTTCCCAA-3', and LIC12730-R1, 5' -GTACCGGACTGTTAAATCTTGACTTCCAC-3'; LIC12922-F, 5' -CACCGAATCACTCAACAGAGTCATTGC-3', and LIC12922-R1, 5'-GAGGGACCGCAATTTTTAACTGAAGTAG-3'. PCR was performed in a reaction volume of $25 \mu \mathrm{l}$ containing $100 \mathrm{ng}$ genomic DNA, $1 \times$ PCR buffer [20 mM Tris/HCl ( $\mathrm{pH} 8.4$ ), $50 \mathrm{mM} \mathrm{KCl],} 2 \mathrm{mM} \mathrm{MgCl}_{2}$, 20 pmol each specific primer, $200 \mu \mathrm{M}$ each $\mathrm{dNTP}$ and $2.5 \mathrm{U}$ Taq DNA polymerase (Invitrogen). Cycling conditions were: $94{ }^{\circ} \mathrm{C}$ for $5 \mathrm{~min}$, followed by 35 cycles of $94{ }^{\circ} \mathrm{C}$ for $50 \mathrm{~s}, 62{ }^{\circ} \mathrm{C}$ for $50 \mathrm{~s}$ and $72{ }^{\circ} \mathrm{C}$ for $1 \mathrm{~min} 30 \mathrm{~s}$, with a final extension of $7 \mathrm{~min}$ at $72{ }^{\circ} \mathrm{C}$. PCR-amplified products were loaded on a $1 \%$ agarose gel for electrophoresis and visualization by ethidium bromide staining.

RNA extraction and RT-PCR analysis. For RT-PCR, total RNA was isolated by the acid guanidinium thiocyanate/phenol/chloroform method using TRIzol Reagent (Invitrogen) according to the manufacturer's recommendations. One microgram of RNA from each sample was treated with $1 \mathrm{U}$ DNase I (Invitrogen) for $15 \mathrm{~min}$ at room temperature. DNase I was inactivated by the addition of $1 \mu \mathrm{l}$ $25 \mathrm{mM}$ EDTA solution followed by incubation at $65^{\circ} \mathrm{C}$ for $10 \mathrm{~min}$. DNase-treated RNAs were reverse transcribed using a SuperScript III First-Strand Synthesis System for RT-PCR (Invitrogen). One-tenth of the reverse transcription product was amplified in a $25 \mu \mathrm{l}$ reaction mix using oligonucleotide primers LIC10494-F/LIC10494R, LIC12730-F/LIC12730-R or LIC12922-F/LIC12922-F as described above. Sample quantity and integrity were verified by amplification of a 1042 bp 16S rRNA gene fragment using primers 16S-F (5'-CAAGTCAAGCGGAGTAGCAATACTCAGC-3') and 16S-R (5'-GATGGCAACATAAGGTGAGGGTTGC-3').

DNA recombinant techniques. The predicted CDSs LIC10494, LIC12730 and LIC12922 were amplified by PCR from total $L$. interrogans serovar Copenhageni strain Fiocruz L1-130 genomic DNA using the primer pairs LIC10494-F and LIC10494-R2 (5'-ACTTTGAGAGCTTCGTCTCGT-3'); LIC12730-F and LIC12730-R2 (5'TCTTGCGAATGAGTTGATCC-3'); LIC12922-F and LIC12922-R2 $\left(5^{\prime}\right.$-ATCAATCTAAATGAAACGTCTCTTC-3'). The PCR product 
was cloned into pENTR-TOPO vector (Invitrogen) followed by transfer/recombination of the DNA insert into the Escherichia coli expression vector pDEST17 (Invitrogen) using LR Clonase (Invitrogen). The vector pDEST17 allows the expression of recombinant proteins with a $6 \times \mathrm{His}$ tag at the $\mathrm{N}$-terminus. All cloned sequences were confirmed by DNA sequencing in an ABI 3100 automatic sequencer (PE Applied Biosystems).

Expression and purification of recombinant proteins. Protein expression was achieved in E. coli strain BL21-SI by the action of T7 DNA polymerase under control of the osmotically induced promoter proU (Bhandari \& Gowrishankar, 1997). E. coli BL21-SI containing recombinant plasmids was grown at $30{ }^{\circ} \mathrm{C}$ in Luria-Bertani broth without $\mathrm{NaCl}$ and with $100 \mu \mathrm{g}$ ampicillin $\mathrm{ml}^{-1}$ with continuous shaking until an $\mathrm{OD}_{600}$ of $0.6-0.8$ was reached. Recombinant protein synthesis was induced by the addition of $150 \mathrm{mM} \mathrm{NaCl}$. After $3 \mathrm{~h}$, the cells were harvested by centrifugation and the bacterial pellets were resuspended in lysis buffer (PBS, pH 7.4, with $100 \mu \mathrm{g}$ lysozyme $\mathrm{ml}^{-1}$ and $2 \mathrm{mM}$ PMSF). The bacterial cell pellets were lysed on ice with the aid of a sonicator (Ultrasonic Processor; GE Healthcare). The soluble fraction was recovered $(10000 \mathrm{~g}, 10 \mathrm{~min})$, the protein was then purified by metal-chelating chromatography in a Sepharose fast flow column (GE Healthcare) and the fractions were analysed by $12 \%$ SDS-PAGE. The purified recombinant protein was dialysed extensively against $0.1 \%(\mathrm{w} / \mathrm{v})$ glycine solution in PBS at a concentration of $10 \mathrm{ml}$ protein per $1000 \mathrm{ml}$ buffer, with at least five changes of buffer every $4 \mathrm{~h}$ for $48 \mathrm{~h}$. Densitometry analysis of the SDS-PAGE gel containing the purified proteins was performed by using Gelquant, version 2.7.0 (DNR Bio-Imaging Systems).

Circular dichroism (CD) spectroscopy. Purified recombinant proteins were dialysed against sodium phosphate buffer ( $\mathrm{pH}$ 7.4). $\mathrm{CD}$ spectroscopy measurements were performed at $20{ }^{\circ} \mathrm{C}$ using a Jasco J-810 spectropolarimeter (Japan Spectroscopic) equipped with a Peltier unit for temperature control. Far-UV CD spectra were measured using a $1 \mathrm{~mm}$ path length cell at $0.5 \mathrm{~nm}$ intervals. The spectra were presented as the mean of three scans recorded from 180 to $260 \mathrm{~nm}$. Spectra data were submitted to Dicroprot software (http://dicroprot-pbil.ibcp.fr), using the K2D method for secondary structure content estimation (Andrade et al., 1993; Deléage \& Geourjon, 1993).

Antiserum. Ten female BALB/c mice (4-6 weeks old) were immunized subcutaneously with $10 \mu \mathrm{g}$ recombinant protein adsorbed to $10 \%(\mathrm{v} / \mathrm{v})$ Alhydrogel $\left[2 \% \mathrm{Al}(\mathrm{OH})_{3}\right.$; Brenntag Biosector], used as adjuvant. Two subsequent booster injections were given at 2-week intervals with the same preparation of $10 \mu \mathrm{g}$ recombinant protein. Negative-control mice were injected with PBS in $10 \%$ Alhydrogel. One week after each immunization, the mice were bled from the retro-orbital plexus and the pooled sera were analysed by ELISA for determination of antibody titres.

Immunoblotting assay. Aliquots $(1 \mu \mathrm{g})$ of each recombinant protein were subjected to SDS-PAGE and then transferred to nitrocellulose membranes (Hybond ECL; GE Healthcare). Membranes were blocked with $10 \%$ non-fat dried milk in PBS containing $0.05 \%$ Tween 20 (PBS-T) and then incubated with antirLIC10494, anti-rLIC12730 or anti-rLIC12922 mouse serum, all at $1: 20000$ dilution in 5\% non-fat dried milk/PBS-T for $2 \mathrm{~h}$ at room temperature. After washing, the membrane was incubated with horseradish peroxidase (HRP)-conjugated anti-mouse IgG (1:5000; Sigma) in $5 \%$ non-fat dried milk/PBS-T for $1 \mathrm{~h}$. The bands were revealed with ECL reagent kit chemiluminescence substrate (GE Healthcare).

ELISA for detection of hamster IgG antibodies. Hamster IgG antibodies against recombinant proteins were detected by ELISA. The plates were covered with $250 \mathrm{ng}$ of each protein per well. All samples were diluted $1: 100$ and evaluated for total IgG using rabbit antihamster IgG antibodies (1:5000; Sigma) and an HRP-conjugated goat anti-rabbit antibody (1:5000; Sigma). The cut-off points were set at $3 \mathrm{SD}$ above the mean $A_{492}$ for sera from six hamsters that had not been exposed to leptospires.

Hamster immunization and challenge studies. Fourteen male Golden Syrian hamsters (6-8 weeks old) were immunized subcutaneously with $50 \mu \mathrm{g}$ recombinant protein adsorbed to $10 \%$ Alhydrogel, used as an adjuvant. Two subsequent booster injections were given at 2-week intervals with the same preparation of recombinant protein $(50 \mu \mathrm{g})$. Negative-control hamsters were injected with $10 \%$ Alhydrogel in PBS. As a positive control, a group of hamsters was immunized with killed whole leptospires (bacterin vaccine), prepared as described by Silva et al. (2007). In brief, washed pellets of L. interrogans serovar Copenhageni strain Fiocruz L1-130 cultures were heat-inactivated at $56{ }^{\circ} \mathrm{C}$ for $20 \mathrm{~min}$, resuspended in PBS and stored at $-20{ }^{\circ} \mathrm{C}$ until use. Hamsters were immunized with a dose of $10^{9}$ inactivated leptospires in $10 \%$ Alhydrogel on days 0 and 14. Two weeks after each immunization, two hamsters of each group were bled by cardiac puncture and the sera were analysed by ELISA for determination of antibody titres. The reciprocal ELISA titre was considered to be the last dilution of serum that registered an absorbance value of 0.10 . Two weeks after the second immunization, groups of seven to ten hamsters were challenged with an intraperitoneal inoculum of $2 \times 10^{5}$ leptospires ( 100 times the $50 \%$ lethal dose) from the same L. interrogans strain, but which had been passaged through Golden Syrian hamsters. Hamsters were monitored daily for clinical signs of leptospirosis and euthanized when clinical signs of terminal disease appeared.

Immunohistochemistry assay. Immunohistochemistry studies were performed to assess the presence of leptospiral antigen (LAg). The primary serum used for the detection of LAg was raised in rabbits according to a previously described procedure (De Brito et al., 1992). Kidney and liver sections of $3 \mu \mathrm{m}$ were analysed using Dako EnVision-based immunohistochemistry methods. After rehydration, the tissue Pro-Bond Plus slides were heated three times for $5 \mathrm{~min}$ in $10 \mathrm{mM}$ citrate buffer in a microwave oven. The sections were then cooled and immersed in $10 \% \mathrm{H}_{2} \mathrm{O}_{2}$ for 15 min to inhibit endogenous peroxidase activity. To block non-specific antigen sites, sections were incubated in PBS with $10 \%$ non-fat dried milk for $30 \mathrm{~min}$ at room temperature. The slides were then incubated with the above primary polyclonal rabbit antiserum diluted $1: 7000$ for $1 \mathrm{~h}$ at room temperature. After several washes with PBS, slides were incubated with polymer-HRP-labelled anti-rabbit antibody (EnVision; Dako) for $40 \mathrm{~min}$ at room temperature and again washed with PBS. Diaminobenzidine/hydrogen peroxidase substrate was incubated for 3-5 min to reach the appropriate intensity and slides were rinsed with distilled water to stop the staining reaction. Immunostained sections were counterstained with haematoxylin for $1 \mathrm{~min}$, washed with tap water, rinsed with distilled water and dehydrated in increasing ethanol concentrations followed by xylene (each treatment, $5 \mathrm{~min}$ ). Finally, the slides were mounted with a coverslip in a non-aqueous permanent mounting medium and observed under a Leica photomicroscope.

Ethics of animal experimentation. All animal studies were approved by the Ethic Committees of the Instituto Butantan and the Faculdade de Medicina Veterinaria e Zootecnia, USP, São Paulo, Brazil.

Statistical analysis. Statistical analysis was performed using GraphPad Prism version 3.00, which calculates survival fractions using the product limit or Kaplan-Meier method (Kaplan \& Meier, 1958). A logrank test (equivalent to the Mantel-Haenszel test) was 
used to compare survival curves. All $P$ values were two-tailed and a value of $P<0.05$ was considered to be statistically significant.

\section{RESULTS}

\section{Bioinformatic analysis}

The genes encoding LIC10494, LIC12730 and LIC12922 were identified by analysis of the genome sequence of chromosome I of L. interrogans serovar Copenhageni and each is present as a single copy (Nascimento et al., 2004a, b). The CDSs LIC10494 and LIC12730 are predicted to be OMPs, whilst LIC12922 is predicted to be a periplasmic protein/OMP based on PSORT (Nakai \& Kanehisa, 1991) and CELLO (Yu et al., 2004, 2006). The PFAM and SMART web servers found no putative conserved domain within the LIC10494 CDS, whilst the LIC12730 CDS presented a tetratricopeptide repeat domain and four NHL (NCL-1, HT2A and LIN-41) repeats, and the LIC12922 CDS had a peptidylprolyl isomerase (PrsA) domain. BLAST analysis showed that the LIC10494 CDS had no homology with bacterial proteins, LIC12730 had partial identity (25\%) with other spirochaetal proteins and LIC12922 had $21 \%$ similarity to other bacterial proteins due to the presence of the PrsA domain. The LipoP program predicted LIC10494 to be a lipoprotein, with a cleavage site for signal peptidase II at aa 20-21 (Juncker et al., 2003). The same data were obtained with the SpLip program (Setubal et al., 2006). Similar putative CDSs for LIC10494, LIC12730 and LIC12922 have been found in L. interrogans serovar Lai (99-100\% identity with LA3735, LA0913 and LA0675, respectively; Ren et al., 2003). LIC12730 and LIC12922 CDSs were also identified in the genome sequences of Leptospira borgpetersenii serovar Hardjo-bovis (89-90\% identity with LBL0936 and LBL2545, respectively; Bulach et al., 2006) and of L. biflexa serovar Patoc (37-48\% identity with LEPBI_I1807 and LEPBI_I0186, respectively; Picardeau et al., 2008). LIC10494 CDS was not identified in either L. borgpetersenii (Bulach et al., 2006) or L. biflexa (Picardeau et al., 2008) genome sequences.

\section{Distribution and expression of genes among Leptospira strains}

The presence of the selected genes in six pathogenic strains and one saprophytic strain of Leptospira was examined by PCR with primer pairs designed according to the $L$. interrogans serovar Copenhageni genome sequence. A DNA fragment was amplified by PCR for the three genes in all six strains belonging to the pathogenic species of $L$. interrogans, except for LIC10494, which was not amplified in $L$. interrogans serovar Grippotyphosa (Fig. 1a). No significant amplification product was detected in the non-pathogenic strain Patoc 1 (L. biflexa serovar Patoc) (Fig. 1a). Template integrity was demonstrated by $16 \mathrm{~S}$ rRNA gene amplification. Gene expression by in vitro-cultured leptospires was evaluated by PCR amplification of reverse-transcribed total RNA. The results obtained revealed the presence of
LIC10494, LIC12730 and LIC12922 transcripts in all of the pathogenic strains, except for L. interrogans serovar Grippotyphosa (Fig. 1b). DNA contamination was ruled out as no amplification was observed in the absence of reverse transcriptase. The integrity of the total RNA used in RT-PCR was determined by the presence of a 1042 bp 16S rRNA gene fragment in all samples (Fig. 1b).

(a)

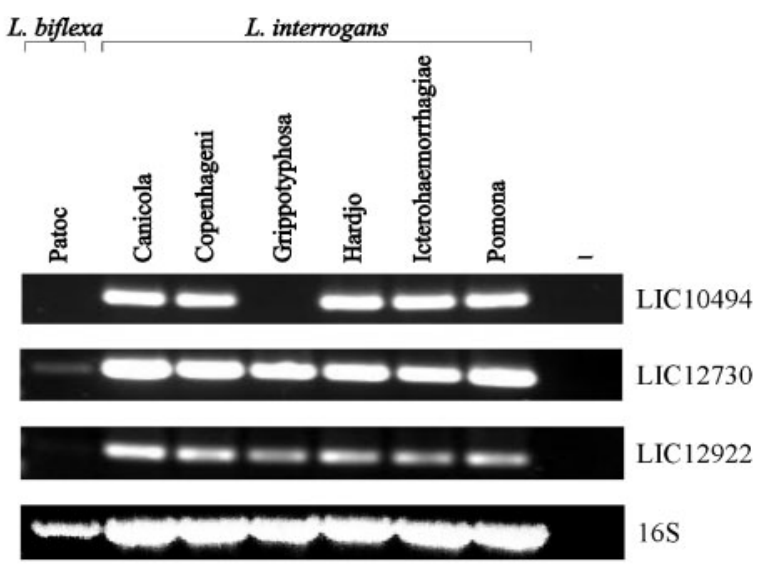

(b)

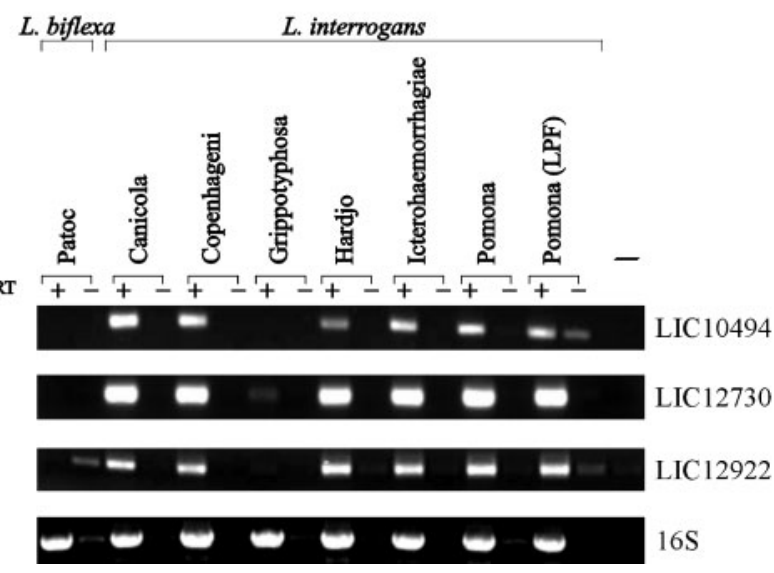

Fig. 1. Distribution and expression of the three CDSs among saprophytic and pathogenic leptospires. (a) Genomic DNA from $L$. biflexa Patoc and six serovars belonging to pathogenic species of $L$. interrogans was subjected to PCR analysis with specific primers designed according to the $L$. interrogans serovar Copenhageni genome sequence. Amplification of the 16S rRNA gene demonstrated template integrity. No DNA was added to the negativecontrol reaction (-). (b) RT-PCR analysis of gene transcripts in high-passage $L$. interrogans strains and low-passage $L$. interrogans serovar Pomona strain LPF. Reactions were performed with the same primer pairs used in (a). Sample quantity and integrity were verified by amplification of a 1042 bp 16S rRNA gene fragment. No DNA was added to the negative-control reaction (-). $\mathrm{RT}+$, Reverse transcriptase present; RT-, reverse transcriptase omitted. 
(a)

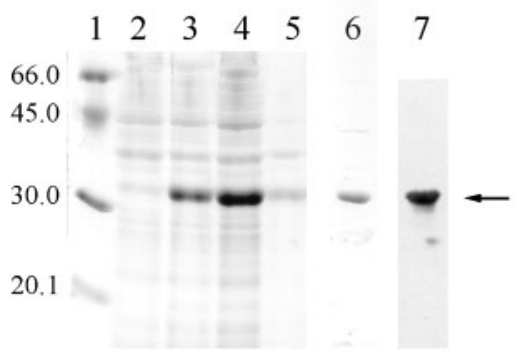

(b)

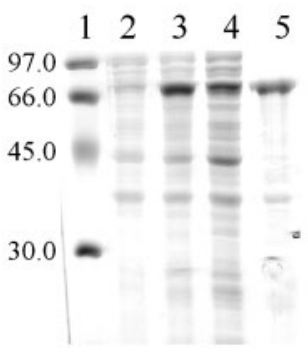

(c)

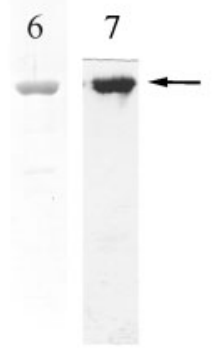

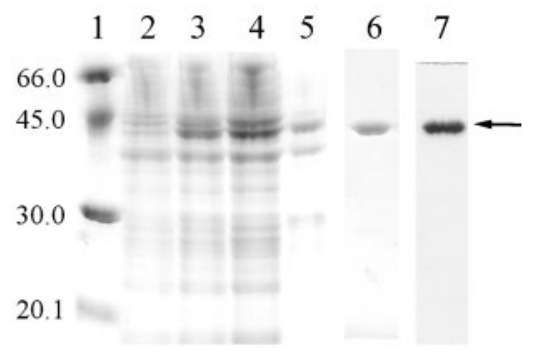

Fig. 2. Analysis of recombinant proteins rLIC10494 (a), rLIC12730 (b) and rLIC12922 (c) from NaCl-induced E. coli BL21-SI by $12 \%$ SDS-PAGE. Lanes: 1 , molecular mass protein marker $(\mathrm{kDa}) ; 2$, uninduced culture; 3, induced culture; 4 and 5 , supernatant and inclusion body pellet after bacterial cell lysis and centrifugation, respectively; 6 , purified protein eluted from $\mathrm{Ni}^{2+}$-charged chelating Sepharose column with $1 \mathrm{M}$ imidazole. Protein bands were visualized by Coomassie blue staining. The proteins were transferred to Hybond ECL membrane and probed with anti-recombinant protein antibodies $(1: 20000)$ and HRP-conjugated anti-lgG $(1: 5000)$ (lane 7). The protein bands were developed with an ECL kit. The expected molecular mass of the recombinant proteins is indicated by an arrow.

\section{Cloning, expression and purification of recombinant proteins}

The genes were amplified, without the signal peptide sequence, and the DNA insert was cloned and expressed as a full-length protein in E. coli. Recombinant proteins were expressed with a $6 \times$ His tag at the $\mathrm{N}$-terminus and purified by metal-chelating chromatography, and an aliquot of each step of the process was analysed by SDS-PAGE (Fig. 2). The recombinant proteins were expressed in E. coli BL21-SI induced cultures with the expected sizes, as evaluated by SDS-PAGE, of 25.8, 77.4 and $40 \mathrm{kDa}$ for rLIC10494, rLIC12730 and rLIC12922, respectively (Fig. 2a-c, lane 3). Although the expression clones of all proteins were fully sequenced, rLIC10494 migrated near the $30 \mathrm{kDa}$ protein standard. The recombinant proteins were purified under native conditions from the supernatants of $E$. coli BL21-SI cultures (Fig. 2a-c, lane 4); the contaminants were washed away with low-concentration imidazole and all proteins were represented as single major bands (Fig. 2a-c, lane 6). By densitometry of the bands, we estimated the purity of each protein to be $92.8,97.5$ and $88.6 \%$ for rLIC10494, rLIC12730 and rLIC12922, respectively. The calculated yield of Luria-Bertani broth culture for each recombinant protein was $3.8,0.75$ and $4.2 \mathrm{mg} \mathrm{l}^{-1}$ for rLIC10494, rLIC12730 and rLIC12922, respectively. Immunoblotting using serum raised in mice against each protein confirmed the integrity of the proteins during expression (Fig. $2 \mathrm{a}-\mathrm{c}$, lane 7).

The secondary structures of the purified recombinant proteins were evaluated by CD spectroscopy. The data from the CD spectra were submitted to Dicroprot and the secondary structure content as calculated by the K2D method is illustrated in Table 1 . The purified recombinant proteins showed a predominance of random and $\beta$-sheet secondary structures in addition to a low percentage of $\alpha$ helical content. The data showed that secondary structures of the three recombinant proteins were maintained after the purification process, rendering them suitable for biological assays.

\section{Reactivity of the recombinant proteins with sera from hamsters experimentally infected with virulent strains of Leptospira}

Sera from 56 hamsters experimentally infected with $L$. interrogans serovar Copenhageni strain Fiocruz L1-130 were evaluated for the presence of total IgG antibodies against each recombinant protein by ELISA at 21 days post-infection (Fig. 3). Sera were considered positive when the mean values at $A_{492}$ were above the mean +3 SD for control sera from six uninfected animals. The results showed a prevalence of total IgG antibodies of 38, 17 and $8 \%$ corresponding to the proteins rLIC10494, rLIC12922 and rLIC12730, respectively.

\section{Antibody response induced by immunization with recombinant proteins}

To assess whether hamsters immunized with the recombinant antigens (rLIC10494, rLIC12730 and rLIC12922) and PBS (control) raised an IgG antibody response against the antigens, sera were collected from the animals

Table 1. Percentage of secondary structure content of each recombinant protein calculated using the Dicroprot software K2D method

\begin{tabular}{|lccc|}
\hline \multirow{2}{*}{ Secondary structure } & \multicolumn{3}{c|}{ Secondary structure content (\%) } \\
\cline { 2 - 4 } & rLIC10494 & rLIC12730 & rLIC12922 \\
\hline$\alpha$-Helix & 5 & 5 & 8 \\
$\beta$-Sheet & 47 & 48 & 43 \\
Random & 48 & 48 & 48 \\
& & & \\
\hline
\end{tabular}




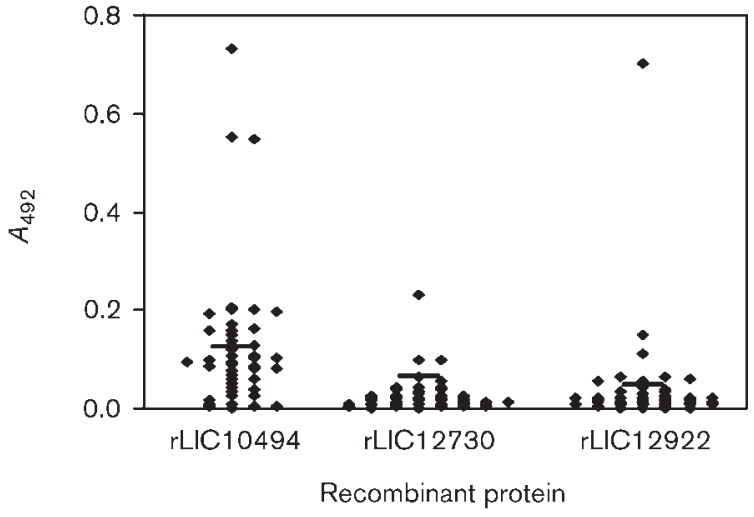

Fig. 3. Recognition of recombinant proteins by $\lg G$ antibodies in sera from hamsters with leptospirosis. Hamsters experimentally infected with $L$. interrogans serovar Copenhageni strain Fiocruz L1-130 were bled and their sera were analysed by ELISA. The cutoff values were defined as the mean +3 SD obtained for sera from six healthy hamsters and are represented by solid lines. prior to the booster (day 15) and prior to (day 30) and after (day 51) the challenge. The animals were bled by cardiac puncture. The antibodies were analysed by ELISA using the recombinant proteins as antigen probes. The data for each protein are shown in Fig. 4(a-c). The results showed that, 2 weeks after the primary immunization (day 15), hamsters produced an antibody response with titres equivalent to $1: 12800,1: 3200$ and $1: 200$ for antirLIC10494, anti-rLIC12730 and anti-rLIC12922, respectively. Two weeks after the second immunization (day 30), the titres increased to $1: 102400,1: 25600$ and $1: 400$, respectively. After challenge (day 51), the titres were $1: 25600,1: 25600$ and $1: 200$, respectively. The booster effect after immunization against all recombinant proteins before (days 15 and 30) and after (day 51) the challenge is shown in Fig. 4(d). No significant level of antibody was detected in control animals immunized with PBS/adjuvant or in animals immunized with bacterin, at any time point. All these data refer to assay 1, shown in Fig. 5(a). (a)

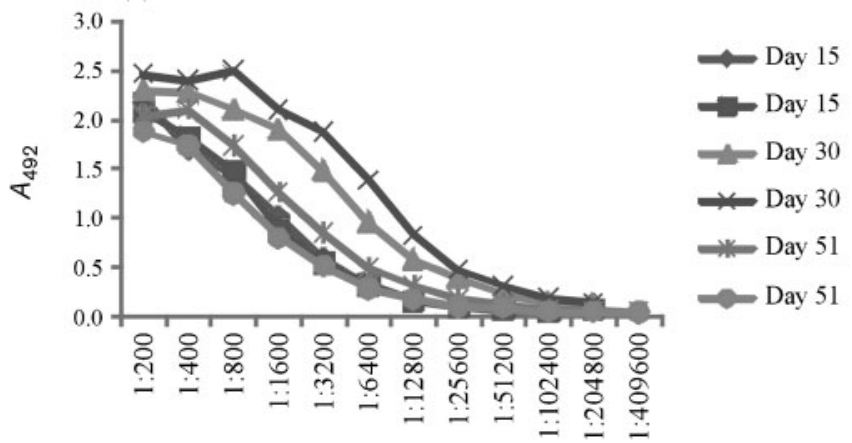

Reciprocal serum dilution

(c)

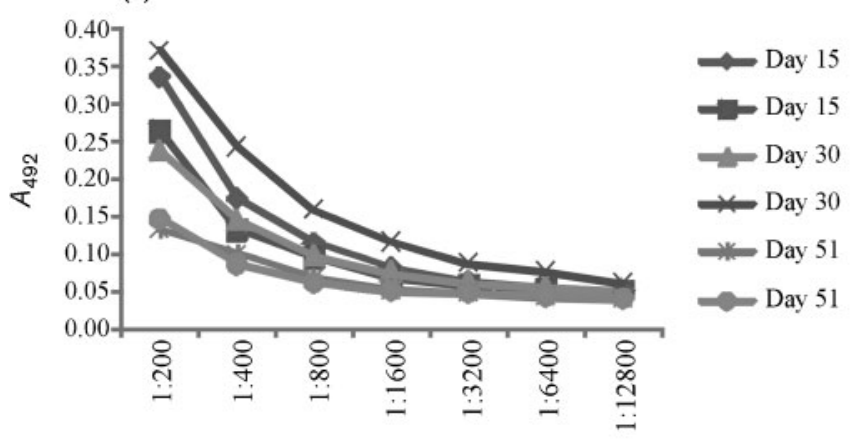

(b)

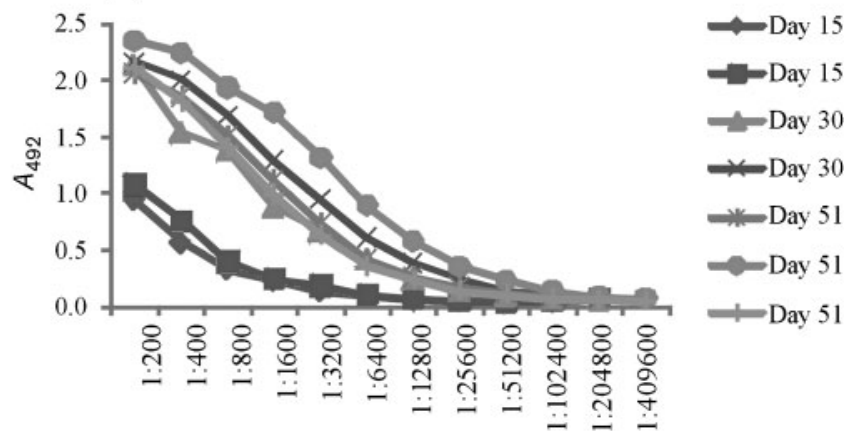

Reciprocal serum dilution

(d)

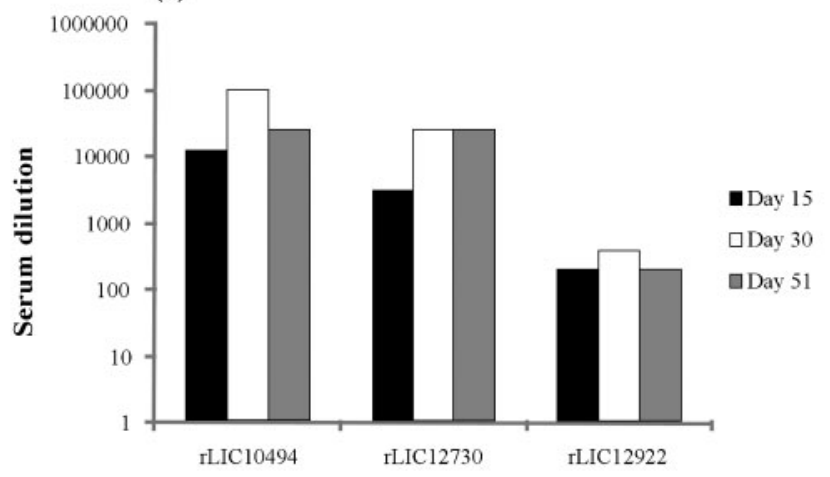

Reciprocal serum dilution

Fig. 4. Induction of a humoral immune response in hamsters. Animals were immunized subcutaneously with PBS (negative control), or rLIC10494, rLIC12730 or rLIC1 2922 (50 $\mu \mathrm{g}$ each). Animals were bled on day 15 (before the booster), day 30 (before challenge) and day 51 (after challenge) to determine antigen-specific total lgG responses in the sera of immunized groups by ELISA. Microdilution plates were coated with rLIC10494 (a), rLIC1 2730 (b) and rLIC12922 (c) and incubated with a serial dilution of serum from the immunized hamsters for $\operatorname{lgG}$ measurements. In (d), the titre values were normalized to those of the serum samples before immunization. The data represent the mean of two hamsters per group (days 15 and 30 ) or survival animals after challenge (day 51). All antibody titres refer to challenge assay 1 (Fig. 5a). 
(a)

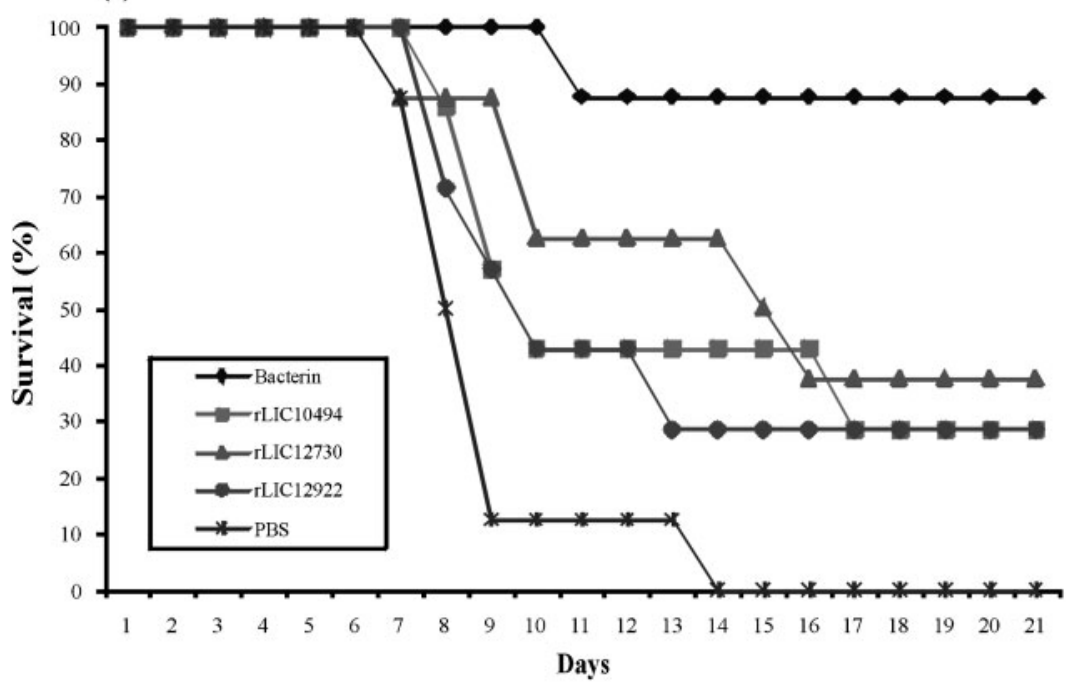

(b)

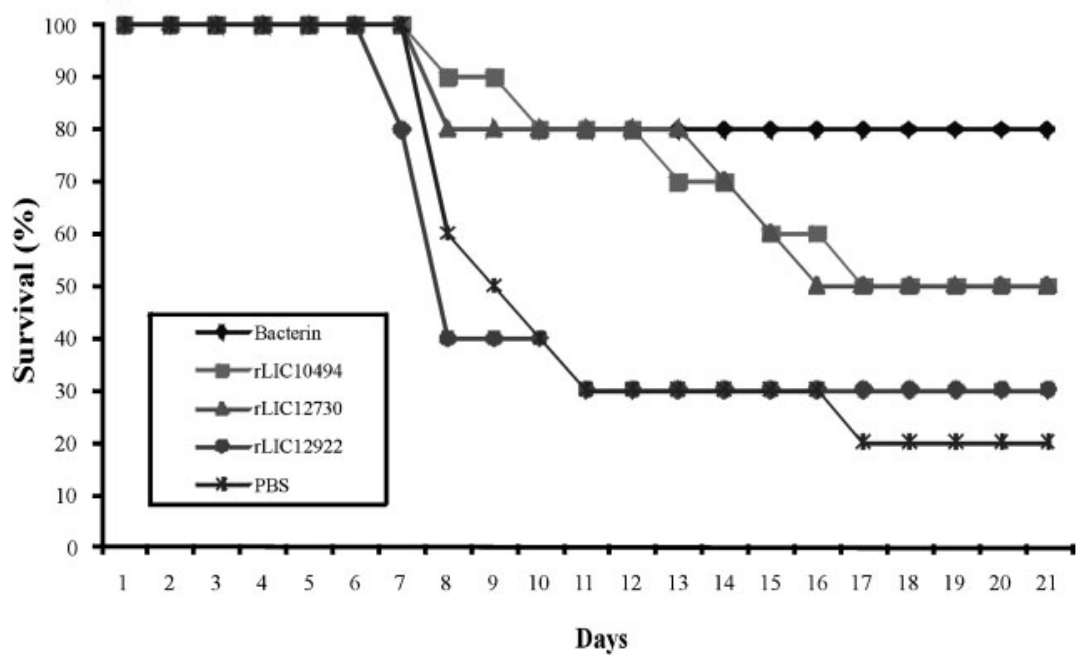

Fig. 5. Survival of hamsters immunized with recombinant proteins and challenged with virulent leptospires. The animals were immunized subcutaneously with PBS (negative control), bacterin (killed whole-leptospires) (positive control), rLIC10494, rLIC12730 or rLIC12922. Two weeks after the second immunization (day 30) with the recombinant proteins, the animals were challenged intraperitoneally with $2 \times 10^{5} \mathrm{~L}$. interrogans serovar Copenhageni strain Fiocruz L1-130 in PBS. The animals were monitored for morbidity until day 21 post-challenge. Data from two independent experiments are shown in (a) and (b).

\section{Protection conferred by hamster immunization with recombinant proteins}

Evaluation of recombinant proteins in the hamster model followed by challenge with virulent leptospires was performed in two independent assays. The survival plots are depicted in Fig. 5 and the data obtained with the statistical analysis are shown in Table 2. Experiment 1 showed that immunization with the rLIC12730 protein conferred partial protection of $38 \%$, followed by $29 \%$ protection for rLIC10494 and rLIC12922, against lethal challenge with $2 \times 10^{5}$ leptospires when compared with the PBS control (Fig. 5a and Table 2). Heat-inactivated wholecell leptospires (bacterin) afforded $88 \%$ protection, whilst no survival was seen with the PBS control group. In the second experiment, $50 \%$ of the animals immunized with the proteins rLIC12730 and rLIC10494 survived, $30 \%$ survived with rLIC12922 and $20 \%$ survived in the PBS control group (Fig. 5b, Table 2). Bacterin afforded $80 \%$ protection. Kaplan-Meier evaluation (Kaplan \& Meier, 1958) of the kinetics of the survival curves showed a statistically significant protective effect for the animals immunized with rLIC12730 and rLIC10494 only with the data of experiment 1 . The surviving animals at day 21 postinfection from experiment 1 were evaluated for the presence of leptospires in their kidneys and histopathology of their kidneys and liver, and the results are summarized in Table 3.

The surviving animals were sacrificed and their kidneys were collected for culture and isolation of leptospires. All hamsters contained leptospires in their kidneys, with the exception of the group vaccinated with the whole-cell preparation, indicating that this bacterin vaccine conferred sterilizing immunity. The histopathological analysis of organs showed significant lesions in almost all animals from the control group, whereas rLIC12730-immunized animals had reduced injuries. The lesions in the kidneys of control animals were characterized as marked acute tubular necrosis and interstitial nephritis, whilst the lesions in the liver were less severe, with inflammatory infiltrates and necrosis (Table 3). The surviving rLIC12730-immunized 
Table 2. Protective efficacy of the recombinant proteins in a hamster model

\begin{tabular}{|c|c|c|c|c|}
\hline \multirow[t]{2}{*}{ Antigen } & \multicolumn{2}{|l|}{ Experiment 1} & \multicolumn{2}{|l|}{ Experiment 2} \\
\hline & \% Protection (no. surviving/total) & $P$ value ${ }^{*}$ & \% Protection (no. surviving/total) & $P$ value $^{\star}$ \\
\hline Killed whole leptospires & $88(7 / 8)$ & 0.0001 & $80(8 / 10)$ & 0.0086 \\
\hline PBS & $0(0 / 8)$ & - & $20(2 / 10)$ & - \\
\hline rLIC10494 & $29(2 / 7)$ & 0.0343 & $50(5 / 10)$ & 0.0852 \\
\hline rLIC12730 & $38(3 / 8)$ & 0.0046 & $50(5 / 10)$ & 0.1157 \\
\hline rLIC12922 & $29(2 / 7)$ & 0.1128 & $30(3 / 10)$ & 0.9524 \\
\hline
\end{tabular}

${ }^{*}$ Statistical analysis was performed using the method of Kaplan \& Meier (1958) and a logrank test to compare survival curves. $P<0.05$ was considered statistically significant (bold).

animals were selected for immunohistochemical analysis of the organs. The data clearly showed LAg deposits in the tissues from animals with severe leptospirosis (Fig. 6b) in contrast to tissues from an uninfected animal (Fig. 6a). An intermediate staining pattern was observed in the tissues of surviving animals from the rLIC12730-immunized group (Fig. 6c-f).

\section{DISCUSSION}

Reverse vaccinology, a genomics-based approach to vaccine development, can overcome conventional methodologies that are time-consuming and allow the identification of only the most abundant protein/antigens (Medini et al., 2008; Serruto \& Rappuoli, 2006). Predicted OMPs have been identified during genome annotation of pathogenic $L$. interrogans serovars Lai (Ren et al., 2003) and Copenhageni (Nascimento et al., 2004a) and L. borgpetersenii (Bulach et al., 2006). The majority are assigned as hypothetical proteins of unknown function, but, due to their putative location, they are believed to play a role in hostpathogen interactions and may be involved in leptospiral pathogenesis and protective immunity. Indeed, Chang et al. (2007) working with putative OMPs of Leptospira that had no similarity to those of other organisms listed in the NCBI database identified novel protein antigens that, although they afforded only partial protection against lethal challenge, could act as a multicomponent vaccine for leptospirosis. More recently, Yan et al. (2010) evaluated a group of putative leptospiral OMPs with an OmpA-like domain that showed protective activity against hamster leptospirosis.

In this work, we have reported the immune response induced by three hypothetical OMPs of unknown function, encoded by the genes LIC10494, LIC12730 and LIC12922. These proteins have been shown experimentally to be surface-exposed in leptospires by live immunofluorescence microscopy (Vieira et al., 2010). DNA amplification was not observed in the non-pathogenic L. biflexa strain for the LIC10494 and LIC12922 genes, and a faint DNA band was observed for the LIC12730 gene. This was expected, as LIC12730 and LIC12922 were only partially identified in the genome sequences of L. biflexa, whilst the LIC10494 gene is absent (Picardeau et al., 2008). All the recombinant proteins were purified in their soluble form, avoiding the use of denaturing agents, and thus retained their structural integrity. Indeed, the expected protein size and integrity of the proteins during expression were confirmed by immunoblotting. CD spectroscopy attested that these proteins maintained their secondary structure after the purification procedure and are therefore suitable for biological assays.

Table 3. Prophylactic efficacy of recombinant antigens evaluated on the basis of culture from organs and histopathology analysis of the challenged surviving animals

\begin{tabular}{|lccc|}
\hline Vaccine & Culture (positive/total) & \multicolumn{2}{c|}{ Histopathology analysis } \\
\cline { 3 - 4 } & & Inflammatory infiltrate in liver & Acute tubular necrosis and interstitial nephritis \\
\hline Killed whole leptospires & $0 / 8(0 \%)$ & $0 / 2(0 \%)^{*}$ & $2 / 2(100 \%)^{*}$ \\
PBS & $2 / 2(100 \%)$ & $1 / 2(50 \%)$ & $2 / 2(100 \%)$ \\
rLIC12730 & $4 / 5(80 \%)$ & $2 / 4(50 \%) \dagger$ & $3 / 4(75 \%) \dagger$ \\
rLIC12922 & $3 / 3(100 \%)$ & $1 / 2(50 \%)^{*}$ & $2 / 2(100 \%)^{*}$ \\
rLIC10494 & $4 / 5(80 \%)$ & $\mathrm{ND}$ & ND \\
\hline
\end{tabular}

ND, Not determined.

*Only two animals were evaluated.

†Only four animals were evaluated. 
(a)
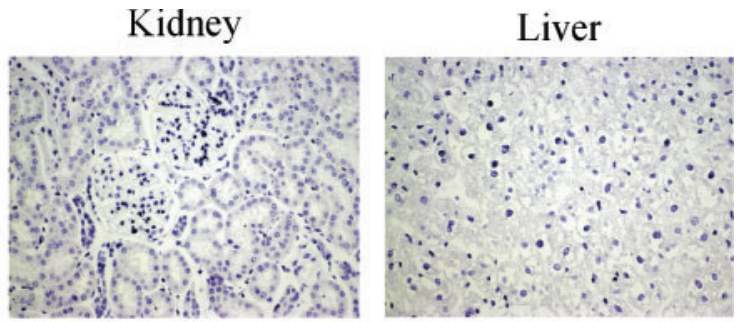

(b)

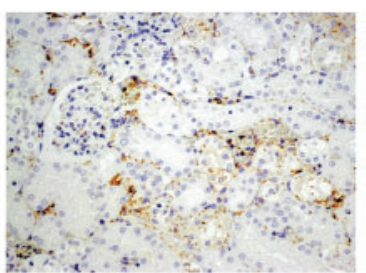

(c)

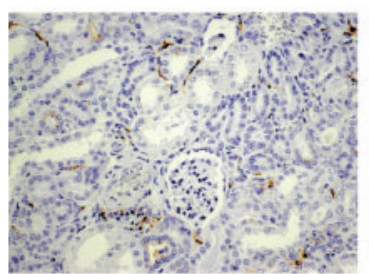

(d)

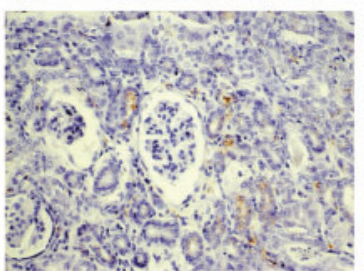

(e)

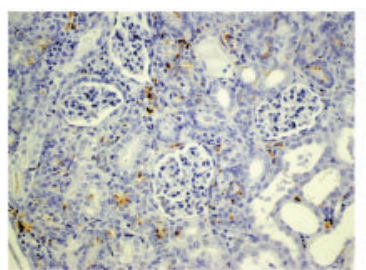

(f)

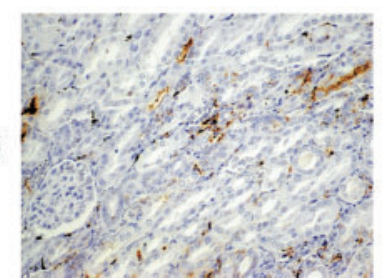

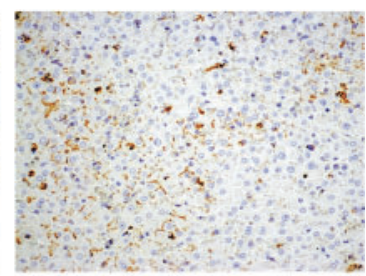
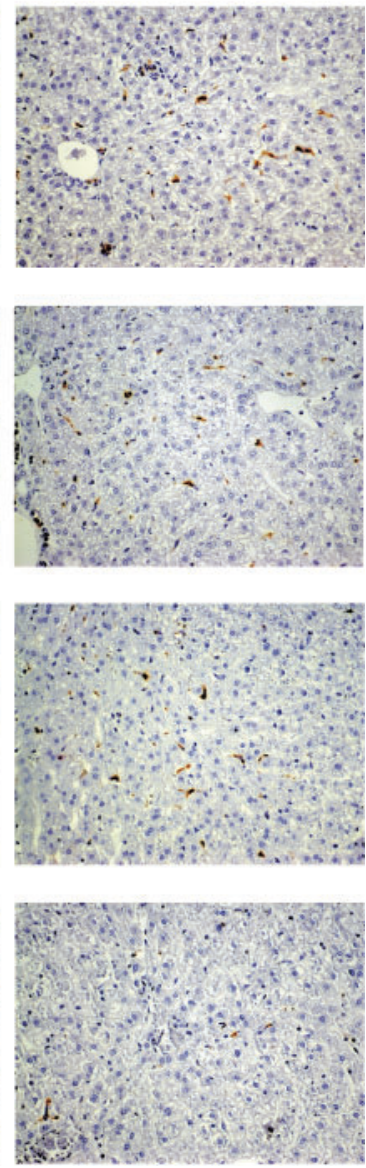

Fig. 6. Immunohistochemistry analysis of organs from challenged hamsters. Kidney and liver tissues obtained from hamsters 21 days after infection with virulent $L$. interrogans serovar Copenhageni strain Fiocruz L1-130 were probed with serum raised against Leptospira total extract ( $\mathrm{LAg})$. (a) No reactivity was observed with anti-LAg in tissues from uninfected animals. (b) LAg reactivity in tissues from experimentally infected animals. (c-f) Tissues from animals immunized with $\mathrm{rLIC} 12730$ showing mild reactivity with anti-LAg. Slides were counterstained with haematoxylin. Magnification $\times 200$.

These proteins are probably expressed during infection because they were recognized by antibodies present in serum samples of hamsters infected experimentally with virulent $L$. interrogans. The protective efficacy of these proteins was analysed in a hamster model using alum as an adjuvant and several parameters were assessed. Our results demonstrated that the rLIC12730 and rLIC10494 proteins afforded partial protection, as revealed by enhanced survival, and reduced histopathological lesions in the liver and kidney in the case of animals immunized with rLIC12730. Kaplan-Meier analysis of the kinetics of the survival curves confirmed a protective, statistically significant effect for the animals immunized with rLIC10494 and rLIC12730 in one of the experiments. The difference in the data obtained with the challenge assays could be attributed to the number of surviving control animals (0/8 vs $2 / 10)$. The displacement of the curves when rLIC12730 or rLIC10494 was administered was indeed interesting and comparable to recent data using OmpA-like domain proteins (Yan et al., 2010). The synergistic immunoprotection efficacy of these proteins in combination or not with other antigens will be further evaluated. In fact, because the LIC10494 CDS is absent in the L. borgpetersenii genome (Bulach et al., 2006), a combination with other leptospiral conserved CDSs will be required. Intriguingly, rLIC10494 was the protein that showed the highest level of specific antibodies in the sera of convalescent hamsters (Fig. 3). Lower levels of antibodies directed against these proteins in human convalescent sera were obtained, ranging from 4 to $7 \%$ (M. V. Atzingen \& A. L. T. O. Nascimento, unpublished results). A protective effect of combined proteins was observed with LipL41-OmpL1 (Haake et al., 1999) and with rLp1454-Lp1118-MCEII (Chang et al., 2007). Preparations using a different adjuvant can also modulate the immune response. Indeed, recent work performed with leptosome-entrapped leptospiral proteins enhanced survival, reduced histopathological lesions in vital organs and reduced leptospiral load in kidneys when compared with the same antigens entrapped with phosphatidylcholine liposomes and E. coli lipid liposomes (Faisal et al., 2009a). In addition, Mycobacterium smegmatis lipids, called smegmosomes, carrying leptospiral antigens were shown to be better adjuvants than alum, as demonstrated by an enhanced antibody response and lymphocyte proliferation, and significant enhancement in both Th1 and Th2 responses (Faisal et al., 2009b).

Survival protection conferred to hamsters immunized with rLIC10494, rLIC12730 and rLIC12922 correlated with the antibody response, suggesting a Th2 immune response. Histopathological analysis further validated our survival data, as animals immunized with rLIC12730 had significantly reduced lesions in their kidneys and liver compared with the control group (Fig. 5 and Table 3). Contrary to the killed whole-leptospire vaccine, the proteins were not able to confer sterilizing immunity, as bacteria could be recovered from the organs of all but one of the rLIC12730-immunized animals (Table 3). However, the 
fact that some of the animals immunized with rLIC12730 and rLIC10494 were clinically normal suggests that the bacterial burden was probably lower when compared with the negative-control PBS group.

In conclusion, we introduced in this work two leptospiral proteins, rLIC12730 and rLIC10494, that showed partial immunoprotection activity against challenge with the homologous strain. These novel antigens may improve the efficacy of a recombinant-based vaccine and may lead to the development of a multicomponent vaccine that could generate cross-protection against a wide range of Leptospira serovars.

\section{ACKNOWLEDGEMENTS}

This work was supported by FAPESP, CNPq and Fundacao Butantan, Brazil. M.V.A. has a post-doctoral fellowship from FAPESP and E. R. A. has a scholarship from PIBIC-CNPq.

\section{REFERENCES}

Adler, B. \& de la Pena Moctezuma, A. (2010). Leptospira and leptospirosis. Vet Microbiol 140, 287-296.

Andrade, M. A., Chacon, P., Merelo, J. J. \& Moran, F. (1993). Evaluation of secondary structure of proteins from UV circular dichroism spectra using an unsupervised learning neural network. Protein Eng 6, 383-390.

Bhandari, P. \& Gowrishankar, J. (1997). An Escherichia coli host strain useful for efficient overproduction of cloned gene products with $\mathrm{NaCl}$ as the inducer. J Bacteriol 179, 4403-4406.

Bharti, A. R., Nally, J. E., Ricaldi, J. N., Matthias, M. A., Diaz, M. M., Lovett, M. A., Levett, P. N., Gilman, R. H., Willig, M. R. \& other authors (2003). Leptospirosis: a zoonotic disease of global importance. Lancet Infect Dis 3, 757-771.

Branger, C., Sonrier, C., Chatrenet, B., Klonjkowski, B., RuvoenClouet, N., Aubert, A., Andre-Fontaine, G. \& Eloit, M. (2001). Identification of the hemolysis-associated protein 1 as a crossprotective immunogen of Leptospira interrogans by adenovirusmediated vaccination. Infect Immun 69, 6831-6838.

Bulach, D. M., Zuerner, R. L., Wilson, P., Seemann, T., McGrath, A., Cullen, P. A., Davis, J., Johnson, M., Kuczek, E. \& other authors (2006). Genome reduction in Leptospira borgpetersenii reflects limited transmission potential. Proc Natl Acad Sci U S A 103, 1456014565.

Chang, Y. F., Chen, C. S., Palaniappan, R. U., He, H., McDonough, S. P., Barr, S. C., Yan, W., Faisal, S. M., Pan, M. J. \& other authors (2007). Immunogenicity of the recombinant leptospiral putative outer membrane proteins as vaccine candidates. Vaccine 25, 81908197.

Cullen, P. A., Haake, D. A., Bulach, D. M., Zuerner, R. L. \& Adler, B. (2003). LipL21 is a novel surface-exposed lipoprotein of pathogenic Leptospira species. Infect Immun 71, 2414-2421.

Cullen, P. A., Xu, X., Matsunaga, J., Sanchez, Y., Ko, A. I., Haake, D. A. \& Adler, B. (2005). Surfaceome of Leptospira spp. Infect Immun 73, 4853-4863.

De Brito, T., Prado, M. J., Negreiros, V. A., Nicastri, A. L., Sakata, E. E., Yasuda, P. H., Santos, R. T. \& Alves, V. A. (1992). Detection of leptospiral antigen ( $L$. interrogans serovar copenhageni serogroup Icterohaemorrhagiae) by immunoelectron microscopy in the liver and kidney of experimentally infected guinea-pigs. Int J Exp Pathol 73, 633-642.

de la Pena-Moctezuma, A., Bulach, D. M., Kalambaheti, T. \& Adler, B. (1999). Comparative analysis of the LPS biosynthetic loci of the genetic subtypes of serovar Hardjo: Leptospira interrogans subtype Hardjoprajitno and Leptospira borgpetersenii subtype Hardjobovis. FEMS Microbiol Lett 177, 319-326.

Deléage, G. \& Geourjon, C. (1993). An interactive graphic program for calculating the secondary structure content of proteins from circular dichroism spectrum. Comput Appl Biosci 9, 197-199.

Faine, S., Adler, B., Bolin, C. \& Perolat, P. (1999). Leptospira and Leptospirosis. Melbourne, Australia: MediSci.

Faisal, S. M., Yan, W., McDonough, S. P., Chang, C. F., Pan, M. J. \& Chang, Y. F. (2009a). Leptosome-entrapped leptospiral antigens conferred significant higher levels of protection than those entrapped with PC-liposomes in a hamster model. Vaccine 27, 6537-6545.

Faisal, S. M., Yan, W., McDonough, S. P., Mohammed, H. O., Divers, T. J. \& Chang, Y. F. (2009b). Immune response and prophylactic efficacy of smegmosomes in a hamster model of leptospirosis. Vaccine 27, 6129-6136.

Finn, R. D., Mistry, J., Schuster-Bockler, B., Griffiths-Jones, S., Hollich, V., Lassmann, T., Moxon, S., Marshall, M., Khanna, A. \& other authors (2006). Pfam: clans, web tools and services. Nucleic Acids Res 34, D247-D251.

Haake, D. A., Mazel, M. K., McCoy, A. M., Milward, F., Chao, G., Matsunaga, J. \& Wagar, E. A. (1999). Leptospiral outer membrane proteins OmpL1 and LipL41 exhibit synergistic immunoprotection. Infect Immun 67, 6572-6582.

Juncker, A. S., Willenbrock, H., Von Heijne, G., Brunak, S., Nielsen, H. \& Krogh, A. (2003). Prediction of lipoprotein signal peptides in Gramnegative bacteria. Protein Sci 12, 1652-1662.

Kaplan, E. L. \& Meier, P. (1958). Nonparametric estimation from incomplete observations. J Am Stat Assoc 53, 457-481.

Ko, A. I., Galvao Reis, M., Ribeiro Dourado, C. M., Johnson, W. D., Jr \& Riley, L. W. (1999). Urban epidemic of severe leptospirosis in Brazil. Salvador Leptospirosis Study Group. Lancet 354, 820-825.

Koizumi, N. \& Watanabe, H. (2004). Leptospiral immunoglobulin-like proteins elicit protective immunity. Vaccine 22, 1545-1552.

Letunic, I., Copley, R. R., Pils, B., Pinkert, S., Schultz, J. \& Bork, P. (2006). SMART 5: domains in the context of genomes and networks. Nucleic Acids Res 34, D257-D260.

Levett, P. N. (2001). Leptospirosis. Clin Microbiol Rev 14, 296-326.

McBride, A. J., Athanazio, D. A., Reis, M. G. \& Ko, A. I. (2005). Leptospirosis. Curr Opin Infect Dis 18, 376-386.

Medini, D., Serruto, D., Parkhill, J., Relman, D. A., Donati, C., Moxon, R., Falkow, S. \& Rappuoli, R. (2008). Microbiology in the post-genomic era. Nat Rev Microbiol 6, 419-430.

Nakai, K. \& Horton, P. (1999). PSORT: a program for detecting sorting signals in proteins and predicting their subcellular localization. Trends Biochem Sci 24, 34-36.

Nakai, K. \& Kanehisa, M. (1991). Expert system for predicting protein localization sites in Gram-negative bacteria. Proteins 11, 95-110.

Nascimento, A. L., Verjovski-Almeida, S., Van Sluys, M. A., MonteiroVitorello, C. B., Camargo, L. E., Digiampietri, L. A., Harstkeerl, R. A., Ho, P. L., Marques, M. V. \& other authors (2004a). Genome features of Leptospira interrogans serovar Copenhageni. Braz J Med Biol Res 37, 459-477.

Nascimento, A. L., Ko, A. I., Martins, E. A., Monteiro-Vitorello, C. B., Ho, P. L., Haake, D. A., Verjovski-Almeida, S., Hartskeerl, R. A., Marques, M. V. \& other authors (2004b). Comparative genomics of 
two Leptospira interrogans serovars reveals novel insights into physiology and pathogenesis. J Bacteriol 186, 2164-2172.

Palaniappan, R. U., McDonough, S. P., Divers, T. J., Chen, C. S., Pan, M. J., Matsumoto, M. \& Chang, Y. F. (2006). Immunoprotection of recombinant leptospiral immunoglobulin-like protein A against Leptospira interrogans serovar Pomona infection. Infect Immun 74, 1745-1750.

Palaniappan, R. U., Ramanujam, S. \& Chang, Y. F. (2007). Leptospirosis: pathogenesis, immunity, and diagnosis. Curr Opin Infect Dis 20, 284-292.

Picardeau, M., Bulach, D. M., Bouchier, C., Zuerner, R. L., Zidane, N., Wilson, P. J., Creno, S., Kuczek, E. S., Bommezzadri, S. \& other authors (2008). Genome sequence of the saprophyte Leptospira biflexa provides insights into the evolution of Leptospira and the pathogenesis of leptospirosis. PLoS One 3, e1607.

Plank, R. \& Dean, D. (2000). Overview of the epidemiology, microbiology, and pathogenesis of Leptospira spp. in humans. Microbes Infect 2, 1265-1276.

Ren, S. X., Fu, G., Jiang, X. G., Zeng, R., Miao, Y. G., Xu, H., Zhang, Y. X., Xiong, H., Lu, G. \& other authors (2003). Unique physiological and pathogenic features of Leptospira interrogans revealed by whole-genome sequencing. Nature 422, 888-893.

Schultz, J., Milpetz, F., Bork, P. \& Ponting, C. P. (1998). SMART, a simple modular architecture research tool: identification of signaling domains. Proc Natl Acad Sci U S A 95, 5857-5864.

Seixas, F. K., Fernandes, C. H., Hartwig, D. D., Conceição, F. R., Aleixo, J. A. \& Dellagostin, O. A. (2007a). Evaluation of different ways of presenting LipL32 to the immune system with the aim of developing a recombinant vaccine against leptospirosis. Can $J$ Microbiol 53, 472-479.

Seixas, F. K., da Silva, E. F., Hartwig, D. D., Cerqueira, G. M., Amaral, M., Fagundes, M. Q., Dossa, R. G. \& Dellagostin, O. A. (2007b). Recombinant Mycobacterium bovis BCG expressing the LipL32 antigen of Leptospira interrogans protects hamsters from challenge. Vaccine 26, 88-95.

Serruto, D. \& Rappuoli, R. (2006). Post-genomic vaccine development. FEBS Lett 580, 2985-2992.

Setubal, J. C., Reis, M., Matsunaga, J. \& Haake, D. A. (2006). Lipoprotein computational prediction in spirochaetal genomes. Microbiology 152, 113-121.

Silva, E. F., Medeiros, M. A., McBride, A. J., Matsunaga, J., Esteves, G. S., Ramos, J. G., Santos, C. S., Croda, J., Homma, A. \& other authors (2007). The terminal portion of leptospiral immunoglobulin-like protein LigA confers protective immunity against lethal infection in the hamster model of leptospirosis. Vaccine 25, 6277-6286.

Turner, L. H. (1970). Leptospirosis. 3. Maintenance, isolation and demonstration of leptospires. Trans R Soc Trop Med Hyg 64, 623-646.

Vieira, M. L., Atzingen, M. V., Oliveira, T. R., Oliveira, R., Andrade, D. M., Vasconcellos, S. A. \& Nascimento, A. L. T. O. (2010). In vitro identification of novel plasminogen-binding receptors of the pathogen Leptospira interrogans. PLoS One 5, e11259.

Yan, W., Faisal, S. M., McDonough, S. P., Divers, T. J., Barr, S. C., Chang, C. F., Pan, M. J. \& Chang, Y. F. (2009). Immunogenicity and protective efficacy of recombinant Leptospira immunoglobulin-like protein B (rLigB) in a hamster challenge model. Microbes Infect 11, 230-237.

Yan, W., Faisal, S. M., McDonough, S. P., Chang, C. F., Pan, M. J., Akey, B. \& Chang, Y. F. (2010). Identification and characterization of OmpA-like proteins as novel vaccine candidates for leptospirosis. Vaccine 28, 2277-2283.

Yu, C. S., Lin, C. J. \& Hwang, J. K. (2004). Predicting subcellular localization of proteins for Gram-negative bacteria by support vector machines based on n-peptide compositions. Protein Sci 13, 14021406.

Yu, C. S., Chen, Y. C., Lu, C. H. \& Hwang, J. K. (2006). Prediction of protein subcellular localization. Proteins 64, 643-651. 